ARTICLE

Received 11 Mar 2014 | Accepted 25 Jul 2014 | Published 12 Sep 2014

DOI: $10.1038 /$ ncomms5827

OPEN

\title{
An assay to image neuronal microtubule dynamics in mice
}

\author{
Tatjana Kleele ${ }^{1}$, Petar Marinković ${ }^{2,3}$, Philip R. Williams ${ }^{1}$, Sina Stern ${ }^{4}$, Emily E. Weigand ${ }^{5}$, Peter Engerer ${ }^{1}$, \\ Ronald Naumann ${ }^{6}$, Jana Hartmann ${ }^{7}$, Rosa M. Karl ${ }^{7}$, Frank Bradke ${ }^{4}$, Derron Bishop ${ }^{5}$, Jochen Herms ${ }^{2,3,8}$, \\ Arthur Konnerth ${ }^{7,8,9}$, Martin Kerschensteiner ${ }^{8,10}$, Leanne Godinho ${ }^{1}$ \& Thomas Misgeld 1 1,3,8,9
}

Microtubule dynamics in neurons play critical roles in physiology, injury and disease and determine microtubule orientation, the cell biological correlate of neurite polarization. Several microtubule binding proteins, including end-binding protein 3 (EB3), specifically bind to the growing plus tip of microtubules. In the past, fluorescently tagged end-binding proteins have revealed microtubule dynamics in vitro and in non-mammalian model organisms. Here, we devise an imaging assay based on transgenic mice expressing yellow fluorescent proteintagged EB3 to study microtubules in intact mammalian neurites. Our approach allows measurement of microtubule dynamics in vivo and ex vivo in peripheral nervous system and central nervous system neurites under physiological conditions and after exposure to microtubule-modifying drugs. We find an increase in dynamic microtubules after injury and in neurodegenerative disease states, before axons show morphological indications of degeneration or regrowth. Thus increased microtubule dynamics might serve as a general indicator of neurite remodelling in health and disease.

\footnotetext{
${ }^{1}$ Institute of Neuronal Cell Biology, Technische Universität München, 80802 Munich, Germany. ${ }^{2}$ Center for Neuropathology and Prion Research, LudwigMaximilians-Universität München, 81377 Munich, Germany. ${ }^{3}$ German Center for Neurodegenerative Diseases (DZNE), 80336 Munich, Germany. ${ }^{4}$ Axon Growth and Regeneration, German Center for Neurodegenerative Diseases (DZNE), 53175 Bonn, Germany. ${ }^{5}$ Department of Physiology, Indiana University School of Medicine-Muncie, Muncie, Indiana 47306, USA. ${ }^{6}$ Transgenic Core Facility, Max-Planck-Institute of Molecular Cell Biology and Genetics, 01307 Dresden, Germany. ${ }^{7}$ Institute of Neuroscience, Technische Universität München, 80802 Munich, Germany. ${ }^{8}$ Munich Cluster for Systems Neurology (SyNergy), 80336 Munich, Germany. ${ }^{9}$ Center of Integrated Protein Science (CIPSM), 81377 Munich, Germany. ${ }^{10}$ Institute of Clinical Neuroimmunology, Ludwig-Maximilians Universität München, 81377 Munich, Germany. Correspondence and requests for materials should be addressed to T.M. (email: thomas.misgeld@lrz.tu-muenchen.de).
} 
T he microtubule cytoskeleton plays a critical role in the establishment and maintenance of neuronal polarization ${ }^{1}$ and is crucial for intracellular transport in axons and dendrites. Contrary to what the term 'skeleton' implies, microtubules are highly dynamic structures constantly being assembled and disassembled ${ }^{2}$. The maintenance of microtubule structure and orientation is hence an important aspect of neuronal homeostasis with loss of microtubules often representing an early stage of neurite dismantling-both during normal development ${ }^{3,4}$ and under pathological conditions ${ }^{5-7}$.

Microtubule dynamics can be assayed using fluorescently tagged end-binding proteins $(\mathrm{EBs})^{8,9}$, a technique that has thus far been applied in vitro and in non-mammalian model systems ${ }^{10-13}$. EB imaging has proven to be an efficient and non-toxic approach to reveal the density and orientation of dynamic microtubules. However, investigating the role of microtubule dynamics in mature mammalian neurons has, to our knowledge, not been possible yet. To assay such dynamics by in vivo imaging in the mammalian nervous system, we generated transgenic mice that express EB3 fused to yellow fluorescent protein (YFP) controlled by neuron-specific Thy1 promoter elements, which have been used previously to generate numerous versatile reporter mice of neuronal morphology, function and organelles ${ }^{14-17}$. When matched by appropriate forms of in vivo microscopy, our approach now allows quantifying microtubule dynamics in a wide range of central and peripheral neurites under physiological conditions, after injury and during chronic neurodegeneration.

\section{Results}

Characterization of Thy1:EB3-YFP transgenic mice. The three Thy1:EB3-YFP transgenic lines (J045, J023, J030) that we characterized in detail showed YFP fluorescence in a broad spectrum of neurons in the central nervous system (CNS) and peripheral nervous system (PNS), but varied in brightness and percentage of expressing cells (Supplementary Fig. 1 and Supplementary Table 1). For all experiments, the mouse line with the lowest expression level and a punctate pattern of fluorescence was used (line J045 for most experiments; J023 for cortical imaging), as strong overexpression of EB3 leads to binding to the entire microtubule lattice $e^{18}$ and pathological swellings in some axons (for example, line J030, which we did not use in our experiments).

To further estimate the level of overexpression of EB3-YFP compared with endogenous EB3, we performed single-cell PCR on EB3-YFP-positive and -negative Purkinje cells, of which only a subset is labelled in line J045. In heterozygous animals of this lowexpressing line, YFP-positive cells show a threefold increase in messenger RNA compared with YFP-negative Purkinje cells $(\Delta \mathrm{Cp}=1.57, n=26$ versus 11 cells per 3 animals). In line with these results, the transgene did not cause any obvious changes of the behaviour, lifespan or fertility of the mice and did not affect neuromuscular morphology, posttranslational tubulin modifications, axonal transport or ultrastructure (Supplementary Figs 2 and 3). Moreover, when we isolated adult dorsal root ganglionic (DRG) and embryonic hippocampal neurons from Thy1:EB3YFP mice (J045 line), we found that the characteristics of transgene-based labelling matched those seen in classical transfection-based assays ${ }^{90}$, that transgene-based labelling colocalized with labelling derived from a spectrally distinct transfected construct, and densities of endogenous accumulations of another EB (EB1) were not affected by the presence of the EB3-YFP transgene (Supplementary Fig. 4). Together, these controls show that in Thy1:EB3-YFP mice expression of the transgene is largely non-toxic and inert as previous established in vitro ${ }^{10,18}$.
Microtubule dynamics in different neuronal compartments. To explore whether Thy1:EB3-YFP mice allowed visualizing dynamic microtubules in intact, mature neurites, we used high-resolution time-lapse microscopy of peripheral axons in intercostal nerves of triangularis sterni explants ${ }^{19}$. We found that the transgenic fusion protein revealed the tips of microtubules as a dynamic pattern of 'comet-like' structures against a faint background of cytoplasmic labelling (Fig. 1 and Supplementary Movie 1). Recordings in the sciatic nerves of anesthetized mice in vivo ${ }^{15}$ showed a similar pattern (Fig. 2a,b and Supplementary Movie 2). Determining the density of these comets ex vivo and in vivo thus provided a direct measure of the local dynamics of the microtubule cytoskeleton in myelinated axons ex vivo $\left(0.065 \pm 0.002\right.$ comets $\mu \mathrm{m}^{-2}$ axonal area in distal motor axons, $n=32$ axons per 8 animals; $0.025 \pm 0.001 \mu \mathrm{m}^{-2}$ in proximal intercostal axons, $n=27 / 6$; mean \pm s.e.m.; for reasoning behind two-dimensional analysis, see Methods) and in vivo in the sciatic nerve $\left(0.042 \pm 0.005 \mu \mathrm{m}^{-2}\right.$, measured at mid-thigh level, an intermediate proximal-distal position, $n=15 / 3$; mean \pm s.e.m.). EB3-YFP comets appeared suddenly, propagated for a distance of a few microns and disappeared (speed: $0.112 \pm 0.003 \mu \mathrm{m} \mathrm{s}^{-1}$ in distal axons ex vivo; $n=32 / 8$; mean \pm s.e.m.). While the speed in mature axons was found to be lower than reported in vitro and in invertebrates (for example, $0.22 \mu \mathrm{m} \mathrm{s}^{-1}$ in hippocampal cultures ${ }^{10}$ ), other comet characteristics, such as orientation and length, are conserved across models. A more detailed analysis of different phases of microtubule growth requires increased imaging frequency $y^{20,21}$, for example, $2 \mathrm{~Hz}$ instead of our routine acquisition rate of $0.5 \mathrm{~Hz}$. As shown previously in vitro ${ }^{20}$, such an imaging regime allows distinguishing growth, pause and reset (likely depolymerization) phases along individual microtubule tracks (Supplementary Fig. 5). However, because high-frequency imaging leads to faster photo-bleaching, we performed the subsequent experiments at $0.5 \mathrm{~Hz}$, which is sufficient to analyse changes in microtubule orientation and density.

To gain further insight into local cytoskeletal properties, we investigated the orientation of dynamic microtubules in different neuronal compartments. In distal intercostal axons, the vast majority $(95 \pm 1 \%, n=32 / 8$; mean \pm s.e.m.) of dynamic microtubules have a 'plus-end out' polarity (Fig. 1); the properties of dynamic microtubules in neuromuscular presynaptic terminals appeared similar, albeit comet density was higher and orientation less stringent (density: $0.118 \pm 0.007 \mu \mathrm{m}^{-2}$; 'plus-end out' orientation: $83 \pm 3 \% ; n=13 / 4$; mean \pm s.e.m.). In contrast, when we imaged microtubule dynamics in proximal Purkinje cell dendrites in acute cerebellar slices, we found a very dense $\left(0.141 \pm 0.011 \mu \mathrm{m}^{-2} ; n=10 / 3\right.$; mean \pm s.e.m. $)$ pattern of comets of mixed orientation ('plus-end out' orientation: $65 \pm 4 \%$; mean \pm s.e.m.; in line with previous reports in cell culture ${ }^{10,22}$; Figure 1b-e and Supplementary Movie 3). To confirm that the reported differences were indeed related to the imaged compartment, rather than a spurious effect of explanting CNS tissue, we devised two-photon imaging assays to measure microtubule dynamics in individual spinal axons and in cortical neurites of live anesthetized mice (Fig. 2a-d, Supplementary Movies 4 and 5). The in vivo density of dynamic microtubules in the spinal cord $\left(0.033 \pm 0.002 \mu \mathrm{m}^{-2} ; n=15 / 3\right.$; mean \pm s.e.m. $)$ was in a similar range to what we measured in the explanted intercostal nerves and in the sciatic nerve in vivo. Also microtubule orientation ('plus-end out': $93 \pm 1 ; n=15 / 3$; mean \pm s.e.m.) and comet speed $\left(0.147 \pm 0.004 \mu \mathrm{m} \mathrm{s}^{-1} ; n=15 /\right.$ 3; mean \pm s.e.m.) were comparable to previous measurements. To image microtubule dynamics in vivo in the brain, we implanted chronic cranial windows ${ }^{23,24}$, which allowed acquiring time-lapse movies of neurons from the somatosensory cortex in adult Thy1:EB3-YFP transgenic mice (Line J023). Despite movement 
a

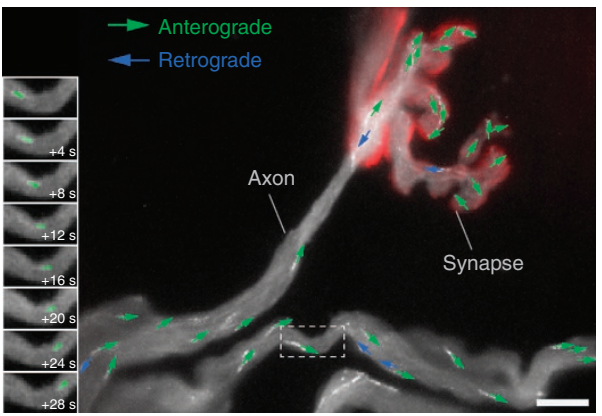

d

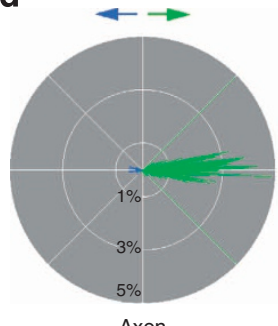

Axon

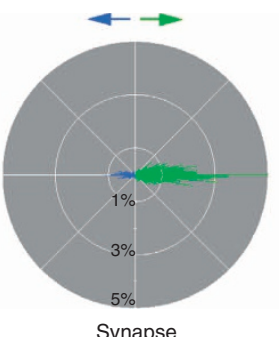

Synapse b
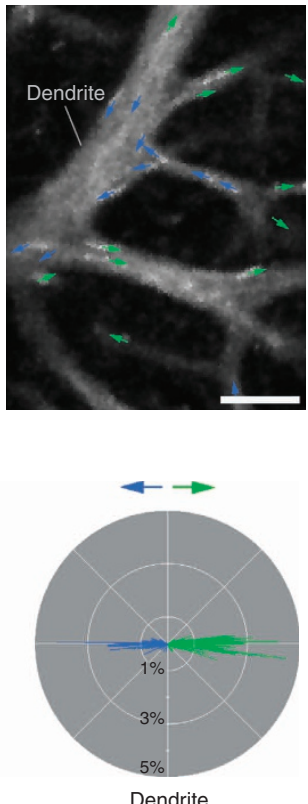

c

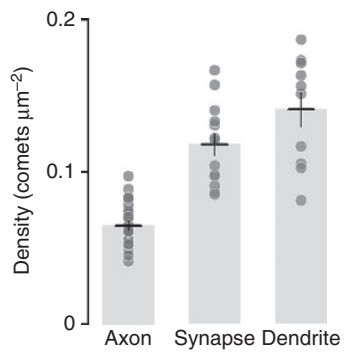

e

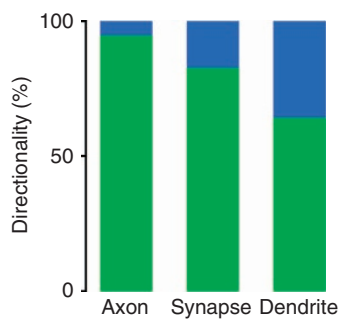

Figure 1 | Imaging microtubule dynamics in Thy1:EB3-YFP mice. (a) A neuromuscular junction (postsynaptic receptors labelled with fluorescently tagged Bungarotoxin, red) and terminal motor axons in an acute Thy1:EB3-YFP triangularis sterni explant. Inset shows eight frames cropped (dashed box in main panel) from a time-lapse sequence of this area highlighting an anterogradely directed EB3 comet (pseudocolored in green). The main panel shows a maximum intensity projection of $20 \mathrm{~s}$ within the time lapse (for full movie, see Supplementary Movie 1 online), where each comet is represented by a colour-coded arrow (anterograde, green; retrograde, blue). (b) Dendrite of a Purkinje cell in an acute cerebellar slice represented in the same way as in (a) (see Supplementary Movie 3). Note the more frequent occurrence of retrogradely directed comets. (c) Comet density in different neuronal compartments ( $n \geq 10$ neurites from $\geq 3$ mice for each group; horizontal line shows mean \pm s.e.m.). (d) Polar plots (that is frequency histograms with angular orientation shown in a circle) of comet directionality in relation to neurite orientation (anterograde to right) in motor axons, neuromuscular synapse terminals and Purkinje cell dendrites ( $n \geq 9$ neurites from $\geq 3$ mice for each group). (e) Mean frequency of comet orientations colour-binned for anterograde and retrograde orientations ( $n \geq 10$ neurites from $\geq 3$ mice for each group). Scale bar in $\mathbf{a}, \mathbf{b}, 5 \mu \mathrm{m}$.

artifacts and reduced signal-to-noise levels in these recordings, we were able to measure density $\left(0.222 \pm 0.017 \mu \mathrm{m}^{-2} ; n=18 / 5\right)$, speed $\left(0.101 \pm 0.004 \mu \mathrm{m} \mathrm{s}^{-1} ; \quad n=18 / 5\right)$ and predominant orientation ( $73 \pm 4 ; n=18 / 5$; all mean \pm s.e.m.) of dynamic microtubules in individual cortical neurites (Fig. 2e and Supplementary Movie 5). Most of the imaged neurites are likely dendritic processes, as confirmed by injection of an adenoassociated virus expressing cytoplasmic CFP, which reveals the presence of spine-like protrusions (Fig. 2e). This in vivo imaging paradigm also allowed longitudinal assessment of microtubule behaviour over periods of 7 days (Fig. 2f). Under physiological conditions, the parameters of dynamic microtubules did not change over time ( $n=8 / 2$; Fig. $2 g-i)$, confirming that our assay provides a robust baseline for future in vivo assessment of microtubule changes in plasticity paradigms. In summary of these in vivo experiments, we can conclude that axons show a lower density of stringently oriented dynamic microtubules compared with dendrites, where densities are higher, but orientation more variable. Moreover, along axons there appears to be a proximalto-distal gradient of increasing density but lower orientation stringency of comets. Finally, most parameters of microtubule dynamics appear insensitive to acute isolation (provided the imaging site stays clear of any axon transection site, see below), stable over time and comparable between PNS and CNS. As the ease of recording, and the stability and quality of measurements are superior in ex vivo nerve-muscle explants compared with the in vivo setting, we used the explant for subsequent experiments.

Microtubule dynamics under pathological conditions. Next, we asked whether our assay could provide useful information in the context of disease-related alterations of the microtubule cytoskeleton. Microtubules play central roles in dynamic cellular processes, such as mitosis, but also under homeostatic conditions, for example by providing stability and support. Both functions of microtubules have been targeted pharmacologically, which comes at risk of substantial side effects. For example, high doses of microtubule-stabilizing drugs, such as paclitaxel ${ }^{25}$, are used as cytostatic agents in oncology, but their application is limited by the risk of inducing toxic neuropathies ${ }^{26}$. Paradoxically, at low doses, paclitaxel and related drugs are also considered as potential therapeutic agents to promote axon survival, as microtubule stabilization might protect axons after trauma ${ }^{27,28}$ and against degeneration $^{6,7,29}$. Obviously, the double-edged effect of microtubule-stabilizing drugs on axons means that it is paramount to devise precise dosing schemes and understand their cell biological consequences. Hence, an assay that allows direct monitoring of the effects of microtubule-stabilizing drugs would be of considerable use. To explore this, we performed dose-response studies to see at which concentration paclitaxel affects dynamic microtubules in intact myelinated axons. Timelapse recordings of Thy1:EB3-YFP intercostal axons revealed loss of EB3 comets after high paclitaxel doses $\left(\mathrm{EC}_{50}\right.$ at $60 \mathrm{~min} \approx 2 \mu \mathrm{M}$; Fig. 3a,b), suggesting 'freezing' of microtubule dynamics. In contrast, at low paclitaxel doses $(\leq 1.5 \mu \mathrm{M})$ EB3 comets were partially preserved for at least $3 \mathrm{~h}$. To probe whether such in situ dosing would allow predictions about outcomes that favoured axon preservation or loss, we explored the effects of different paclitaxel doses on acute axonal degeneration $\left(\mathrm{AAD}^{30}\right)$. AAD removes parts of severed axons within an hour of axotomy and has been previously identified as a possible target for low-dose 


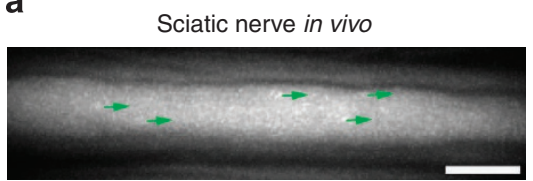

b

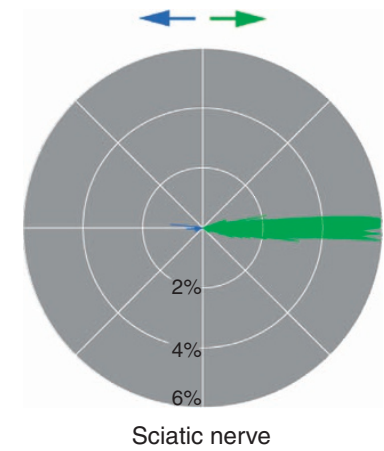

C

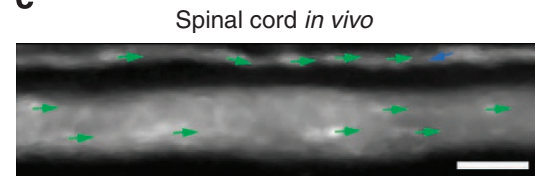

d

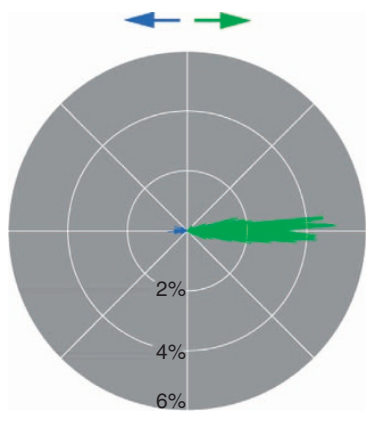

Spinal cord e

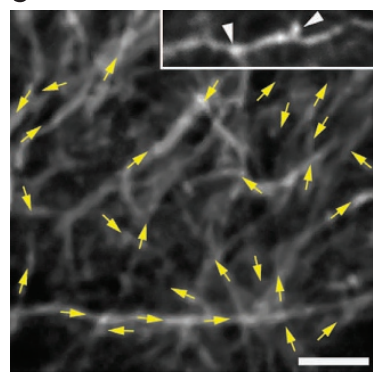

g

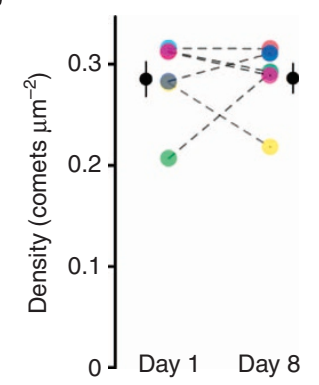

f
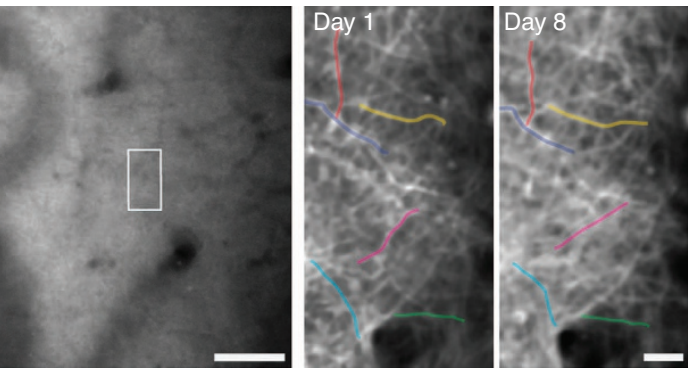

h

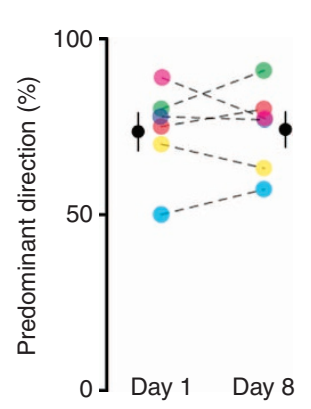

i

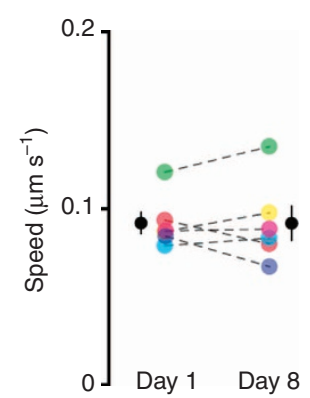

Figure 2 | In vivo imaging of microtubule dynamics in the PNS and CNS. (a) Microtubule dynamics in a sciatic nerve axon of an anesthetized Thy1:EB3-YFP mouse (image represented as in Fig. 1; see Supplementary Movie 2). (b) Polar plot shows comet directionality of axons in the sciatic nerve. (c) Microtubule dynamics in an axon in the lumbar spinal cord in vivo (see Supplementary Movie 4). (d) Polar plot of axons in lumbar spinal cord.

(e) Neurites of the somatosensory cortex imaged through a cranial window in vivo (see Supplementary Movie 5). Comets are colour coded in yellow, as anterograde and retrograde cannot always be defined in this preparation. Inset shows CFP channel of another mouse that was injected with a CFPexpressing adeno-associated virus to illustrate spine-like protrusions (arrowheads) on EB3-measured neurites. (f) Chronic imaging of the same neurites in the somatosensory cortex in vivo over 1 week. A larger overview of the cranial window (left) allows the same six neurites to be identified on day 1 and 8 (boxed on the left; neurites pseudocolored on the right). (g,h,i) Density, predominant direction and speed were measured in the same six neurites (identified by colour and linked by dashed line; black data symbol shows mean \pm s.e.m.) as shown in (f) on day 1 and day 8 . Scale bar in a,b,e,f (higher magnification panels and inset) $5 \mu \mathrm{m}$ and in $\mathbf{f}$ (overview) $50 \mu \mathrm{m}$.

paclitaxel therapy ${ }^{27}$. Indeed, when we measured the extent of AAD-mediated axonal dieback after two-photon laser cuts of motor axons, we found a significant protection of the axons at low doses $(1.5 \mu \mathrm{M})$ of paclitaxel (Fig. $3 \mathrm{c}, \mathrm{d})$, while high paclitaxel doses $(10 \mu \mathrm{M})$ exacerbated axonal dieback.

Previous studies in invertebrate neurons have suggested that axotomy has drastic acute and chronic effects on microtubule dynamics ${ }^{11,31}$. To what degree such findings also hold true in mature mammalian axons remains unclear, as non-mammalian axons differ substantially from mammalian ones in their behaviour after injury ${ }^{32,33}$. Our assay revealed that immediately
$(<5 \mathrm{~min})$ after laser transection of motor axons ${ }^{34}$, a greater than fourfold increase in dynamic microtubule density was observed compared with non-severed axons (at $0-10 \mu \mathrm{m}$ from the axonend: $0.283 \pm 0.032 \mu \mathrm{m}^{-2}$ axonal area; $n=9 / 7$; mean \pm s.e.m.; Fig. 4a,b, Supplementary Movie 6). This increase was a local phenomenon, as comet density remained normal $60-70 \mu \mathrm{m}$ proximal to the injury site. The polarity of comets in the area proximal to the cut was largely unaltered, with the majority of the excess comets propagating anterogradely, although the orientation of individual comets paralleled the axonal axis less strictly near the severed end than further away. While our results 
a
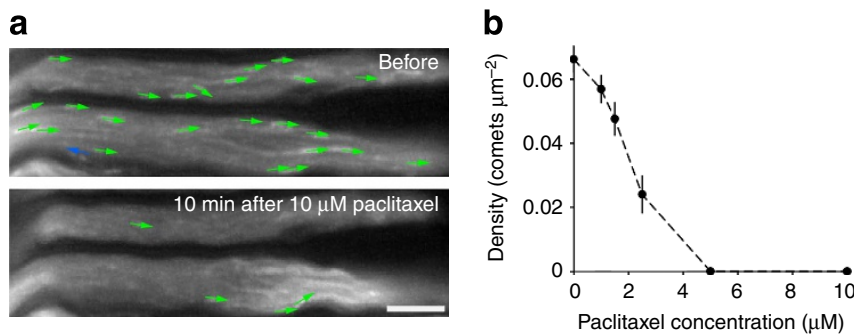

C

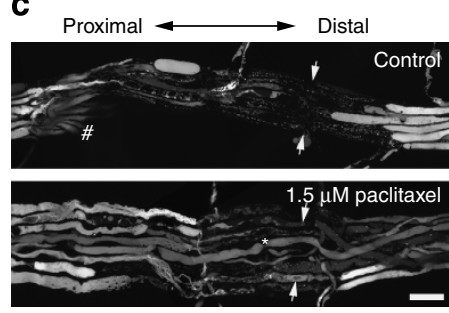

d

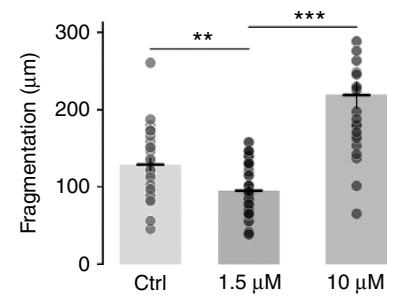

Figure 3 | Microtubule dynamics after exposure to paclitaxel. $(\mathbf{a}, \mathbf{b})$ The microtubule-stabilizing drug paclitaxel leads to a dose-dependent loss of comets in motor axons ex vivo. (a) shows time-lapse analysis represented as in Fig. 1; in (b), the corresponding dose-response curve measured $60 \mathrm{~min}$ after paclitaxel application ( $n \geq 11$ axons from 3 mice for each group; mean \pm s.e.m.). (c,d) Low-dose paclitaxel protects against acute axonal degeneration (AAD). (c) Shows confocal micrographs of axon fascicles in explants from Thy1:YFP-16 mice (all motor axons are labelled), $1 \mathrm{~h}$ after several axons were severed by two-photon microsurgery (arrows) in untreated (top) and paclitaxel-pretreated $(1.5 \mu \mathrm{M}$ for $1.5 \mathrm{~h}$ before and for $1 \mathrm{~h}$ after axotomy; bottom) explants. Note dieback marked by trails of axon fragments. Some axons could not be severed as they lay too deep (asterisk); other axons leave the nerve in a fascicle (pound symbol); both were not included in the analysis. (d) Shows a quantification of the AADmediated dieback distance in untreated control, 1.5 and $10 \mu \mathrm{M}$ paclitaxeltreated axons ( $n \geq 25$ axons from 3 mice per group horizontal line shows mean \pm s.e.m.). Scale bar in $\mathbf{a} 5 \mu \mathrm{m}$ and in c $20 \mu \mathrm{m}$. ${ }^{\star \star \star} P<0.001$; ${ }^{\star \star} P<0.01$, t-test.

confirm acute injury-induced changes in microtubule dynamics, we did not observe 'traps' of microtubules that are directed in opposing directions as described in transected axons of isolated Aplysia neurons ${ }^{11}$.

To examine whether more global changes of microtubule dynamics occur at a later stage of the injury response as shown in flies $^{35}$, we mechanically transected intercostal nerves in vivo ${ }^{15}$ and then imaged EB3-YFP dynamics distal and proximal to the lesion between $3 \mathrm{~h}$ and 2 days after injury (Fig. $4 \mathrm{c}-\mathrm{e}$ ). A global proximal increase in comet density started $6 \mathrm{~h}$ after cut and reached a greater than threefold increase within 2 days (at $\sim 1 \mathrm{~mm}$ from the lesion, $6 \mathrm{~h}$ after cut: $0.033 \pm 0.003$ versus $0.023 \pm 0.002 \mu \mathrm{m}^{-2}$ in uncut contralateral control nerves; 2 days after cut: $0.087 \pm 0.006$ versus $0.022 \pm 0.001 \mu \mathrm{m}^{-2} ; n \geq 9 / 2$ for both time points; all mean \pm s.e.m.). After transection, motor axons start to form growing tips to reinnervate their target muscle ${ }^{36}$. When we measured microtubule dynamics in axon tips during regeneration, we detected a significant sustained increase in dynamic microtubule densities ( 9 days after cut: $0.195 \pm 0.020$; $n=15 / 3$; mean \pm s.e.m.; Supplementary Movie 7). Surprisingly, comet density also increased transiently in the distal nerve stump $6 \mathrm{~h}$ after injury, but decreased before Wallerian fragmentation set in (Fig. 4d,e). To ascertain whether this distal increase was part of the Wallerian degeneration programme ${ }^{37}$, even though it preceded overt fragmentation by several hours, we crossed Thy1:EB3-YFP transgenic animals with $\Delta N L S-W l d^{S}$ mice that express a predominantly axonal and therefore particularly potent version (deleted nuclear localization signal; $\Delta \mathrm{NLS}$ ) of the slow Wallerian degeneration $\left(\mathrm{Wld}^{\mathrm{S}}\right)$ protein ${ }^{38}$. In such mice, the distal stump of cut axons remains intact for up to 2 weeks. While the proximal response to axotomy was similar to the wild-type situation, although the increase in comet density was somewhat delayed, the distal stump showed largely unaltered comet densities at all time points examined after injury (up to 3 days; Fig. 4f,g). This suggests that the proximal and the distal increase in comet densities are mechanistically distinct and that changes in microtubule dynamics are immediately linked to the Wallerian degeneration programme in disconnected axon segments. So, on the one hand, increased microtubule dynamics could serve as a tag of destabilized axons that are destined to degenerate. On the other hand, the delayed and sustained increase in microtubule dynamics in the proximal stump correlates with regenerative growth, rather than with degeneration. We therefore wondered, whether microtubule remodelling could also be found in axons that receive growthpromoting stimuli, but were never injured. As the triangularis muscle is intersegmental and receives input from several intercostal nerves ${ }^{19}$, axotomy of one intercostal nerve can induce expansion of motor units in neighbouring nerves, as their axons strive to reinnervate vacated neuromuscular junction sites (Fig. 4c). Indeed, when we assayed microtubule dynamics in neighbouring, non-transected intercostal axons, we found that comet density doubled ( 2 days after cut: $0.042 \pm 0.004 ; n=15 / 3$ versus contralateral side: $0.020 \pm 0.001 ; \quad n=10 / 3 ; \quad$ all mean \pm s.e.m.; Fig. 4e, right). These results suggest that EB3comet density could serve as a general marker of ongoing or imminent remodelling of axonal arbours.

The drastic changes in microtubule dynamics that we found in traumatic forms of axon degeneration in Thy1:EB3-YFP mice raised the question of whether similar changes can be found in chronic neurodegenerative diseases ${ }^{6}$. To investigate this, we crossed Thy1:EB3-YFP mice to mice that overexpress mutant forms of human superoxide dismutase 1 (SOD mice), a widely used model of familial amyotrophic lateral sclerosis. Indeed, in two different strains of SOD mice (for description of mutants, see ref. 39), showing either a very rapid $\left(\mathrm{SOD}^{\mathrm{G} 93 \mathrm{~A}}\right)$ or a more protracted (SOD1 ${ }^{\mathrm{G} 85 \mathrm{R}}$ ) disease course, we found an increase in comet density in otherwise intact looking intercostal axons. In contrast, 4-month-old control mice that overexpressed a nonmutated form of human SOD1 showed normal microtubule dynamics (Fig. 5).

\section{Discussion}

Taken together, our study demonstrates that in vivo imaging of Thy1:EB3-YFP mice allows assaying microtubule dynamics and polarization in different compartments of fully mature mammalian neurons in the PNS and CNS. Thus, the status of the microtubule cytoskeleton can be determined in acute and chronic models of axonal injury, as well as under the influence of microtubule-modifying drugs, which are in wide use in oncology as cytostatic agents where neurotoxicity limits their use $e^{26}$ Moreover, modulating microtubule stability has been suggested as a therapeutic approach to prevent secondary axon loss after trauma, promote regrowth or even halt neurodegeneration ${ }^{7,28}$. Importantly, it is not always clear to what extent such beneficial effects result from direct action on neurons or rather on other cells, such as glia or fibroblasts. As dosing and cell specificity are clearly critical in clinical translation, our neuron-specific in vivo assay will be useful in further developing such approaches in mammals. Finally, increased microtubule dynamics might serve as an early indicator of axon destabilization and plasticity, as it 
a

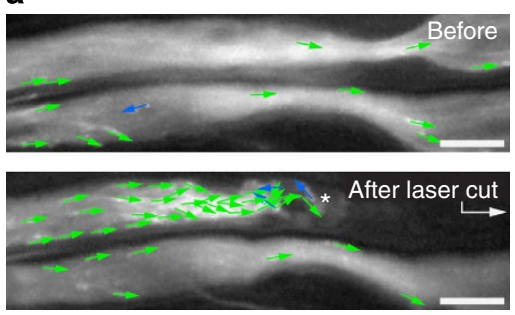

b

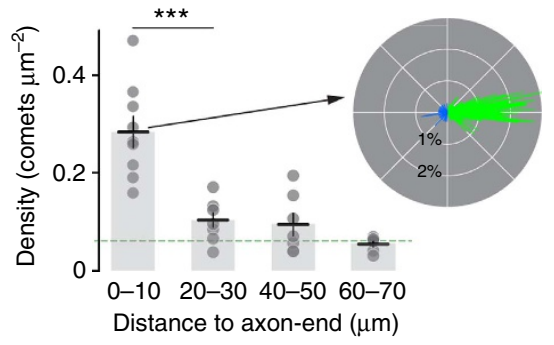

C

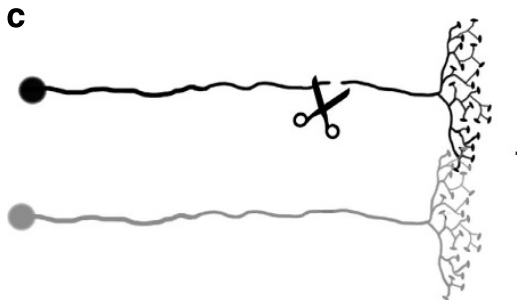

d

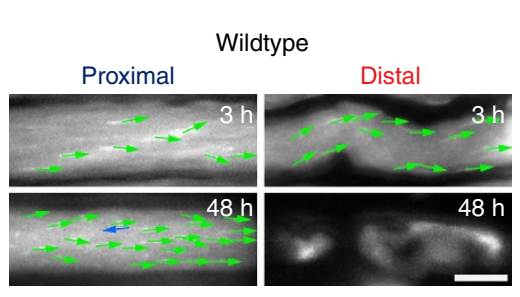

$\mathbf{f}$

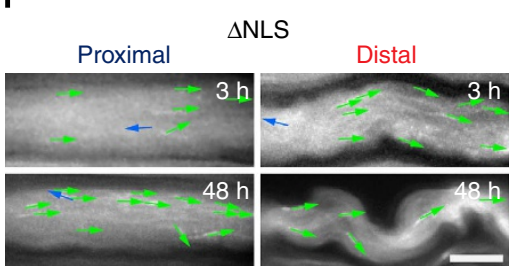

$48 \mathrm{~h}$

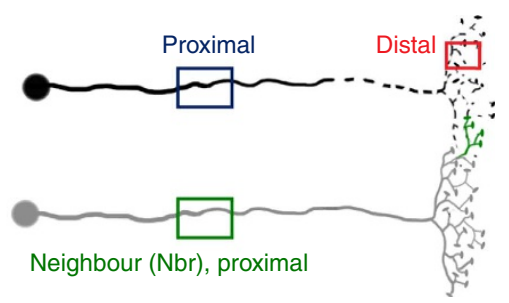

e

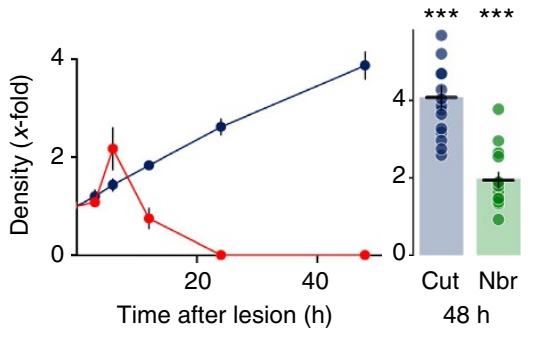

9

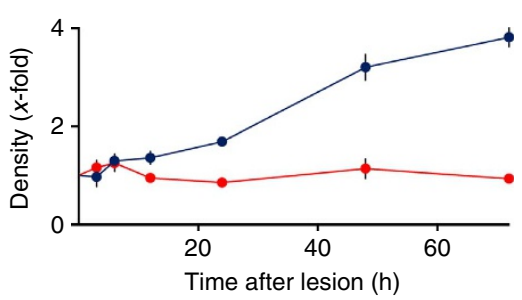

Figure 4 | Microtubule dynamics after acute and chronic injury. (a,b) Acute axotomy by two-photon laser (axotomy site outside of the frame; arrow in a) induces a rapid increase in comet density at the proximal axon-end (asterisk; Supplementary Movie 6). Comet density in the lower axon is unchanged, because the cut is $>60 \mu \mathrm{m}$ outside of the frame. Quantification (b) shows that this effect is confined to within less than $60 \mu \mathrm{m}$ from the axon-end and comet orientation is largely unaltered (horizontal line shows mean \pm s.e.m.; polar plot of the 0-10 $\mu \mathrm{m}$ segment shown as insert; the dashed green line indicates control values; $n \geq 7$ neurites from $\geq 5$ mice for each group; polar plot from four axons from four mice). (c-e) In contrast to the local increase in microtubule dynamics seen acutely after axotomy, global changes in comet density are found within $12 \mathrm{~h}$ after nerve cut (schematic of experiment and measurement sites in c). The 'neighbour' is an uncut adjacent nerve that can sprout into the denervated territory (green sprout symbol on the right in c). Proximal of the cut ( $\sim 1 \mathrm{~mm}$ away) comet density steadily increases, while distally an initial spike in dynamics is followed by a decline before fragmentation (d,e, left). (e) Right shows that axons in the neighbouring nerve that are not cut, but receive a growth stimulus ('Nbr'), also show increased microtubule dynamics after $48 \mathrm{~h}$ ( $n \geq 10$ neurites from $\geq 3$ mice for each condition; bar showing 'cut, $48 \mathrm{~h}$ ' is a re-plot from time course on left; horizontal line and data symbols show mean \pm s.e.m., respectively). (f,g) Changes in microtubule dynamics in the distal axotomized stump are abolished by overexpressing a fusion protein that blocks Wallerian degeneration (' $\Delta \mathrm{NLS}$ '), while the proximal changes are delayed but still present $(n \geq 11$ neurites from $\geq 2$ mice for each group; data symbols indicate mean \pm s.e.m.). In panels e,g some error bars are hidden by the data symbol. Scale bar in all panels $5 \mu$ m. ${ }^{\star \star \star} P<0.001$, analysis of variance.

equally precedes degenerative destruction (in Wallerian degeneration or in amyotrophic lateral sclerosis models), as well as denervation-induced outgrowth. Given that microtubule dynamics also marks a subset of dendritic spines and regulates spine shape in vitro ${ }^{40}$, increased EB3-comet activity could serve as a tag of remodelling neurite segments. Clearly, final proof of this notion will require careful in vivo experiments, for example, using induced remodelling paradigms in the somatosensory cortex ${ }^{41}$ or spontaneous remodelling processes, such as those found in developing motor axons ${ }^{42}$. Importantly, with the new in vivo tools presented here, testing this hypothesis, as well as exploring the role of microtubule remodelling in a broad range of mouse models of neurological diseases is now possible.

Still, before our approach is transferred to new applications, some considerations need to be kept in mind: First, in Thy1:EB3YFP mice, a biologically active fusion protein is overexpressed. As even YFP overexpression alone can have toxic effects in axons ${ }^{43}$, it is important to include appropriate controls in any new setting in which the assay is used. Importantly, in our low-expressing mouse lines overexpression seems moderate, and so far, we did 

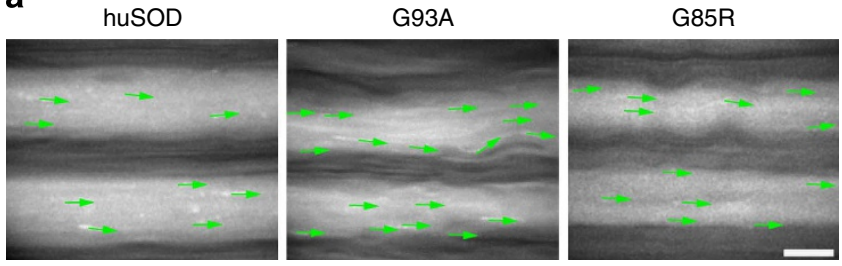

b

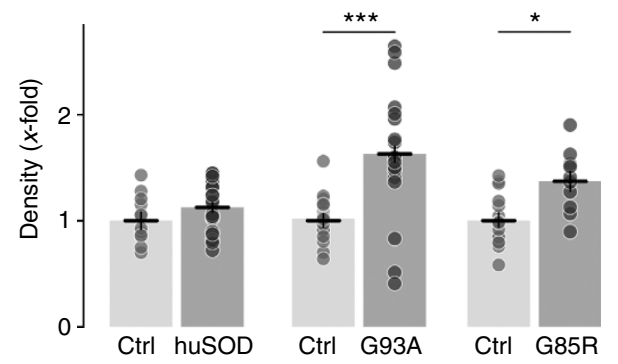

Figure 5 | Microtubule dynamics in neurodegenerative disease.

$(\mathbf{a}, \mathbf{b})$ Microtubule dynamics are altered with increased comet densities in two models of familial ALS, but not in control mice that overexpress a non-mutated form of human SOD1 (Ctrl: respective wild-type litter mates as controls; 'huSOD', non-mutated form of SOD1, P120; 'G93A': SOD1G93A mutation, P120; 'G85R', SOD1 ${ }^{G 85 R}$ mutation, preterminal; $n \geq 10$ neurites from $\geq 2$ mice for each group; data symbols indicate mean \pm s.e.m.). Scale bar in (a) $5 \mu \mathrm{m}$. ${ }^{\star \star \star} P<0.001 ;{ }^{\star} P<0.05$, analysis of variance.

not detect any signs of pathology. Furthermore, for those parameters that can be compared, our assay is in accordance with previous approaches (for example, comet-like versus filamentous distribution; coincidence of transgeneand transfection-based labelling in vitro; microtubule orientation $^{8,10,44}$ ). In addition to general toxicity, specific effects of EB3 overexpression should be considered. For example, EB3 interacts with other plus-end-binding proteins, such as CLIPs ${ }^{18}$. Our construct lacks this interaction domain ${ }^{10}$; however, as the endogenous EB3 locus is intact, only a partial loss of function would be expected-and indeed, in vitro this interference becomes relevant only at high levels of overexpression that coincide with loss of a comet-like staining pattern ${ }^{18}$. Second, CNS in vivo imaging of microtubule dynamics requires careful design of excitation and emission optics of the two-photon systemwhich is not surprising given that the structure imaged here has a size roughly an order of magnitude below the diffraction limit in width (a microtubule's diameter is $\sim 25 \mathrm{~nm}$ ) and which has an intrinsic limitation in 'signal-over-background' if high levels of overexpression are avoided. Therefore, for automated analysis as previously established for EB comets in vitro ${ }^{20}$, even optimally acquired in vivo data sets can prove challenging. Third, while EB3-YFP marks a well-defined structure in cell biological terms, an increase in comet density alone does not directly predict whether the microtubule cytoskeleton undergoes net expansion or contraction, as dynamic microtubules would be expected to be more abundant in both settings. However, microtubular breakdown and expansion are linked to early steps of neurite loss and outgrowth, respectively, so that our assay provides a means to identify sites of imminent neuronal remodelling. Hence, while above-mentioned considerations are important to keep in mind for future applications, the assay described here adds microtubule remodelling to the growing list of cell biological properties of neurons that can be measured in vivo in the mammalian nervous system during development, homeostasis and disease.

\section{Methods}

Animals. For specific labelling of dynamic microtubule tips in neurons, we used regulatory elements of the Thy1-promoter ${ }^{14}$ to express YFP-tagged end-binding protein 3 (EB3) in neurons ${ }^{45}$. For this, an amino-terminal in-frame fusion was generated between the coding sequence of YFP and EB3 (derived from an EB3-GFP vector provided by B. Link, Medical College of Wisconsin, USA). This EB3-YFP fusion gene was cloned into an expression vector downstream of neuron-specific elements of the Thy1-promoter ${ }^{14,46}$. Thy1:EB3-YFP transgenic mice were generated using standard pronuclear injection procedures, and 10 founder lines were screened to establish the three transgenic mouse strains (J045, J023 and J030) described in this study-these are now past the F7 generation and appear to be stable in their expression pattern. J045, the lowest expressing line, was used for most experiments. Parameters of neuromuscular health were determined in J045, as well as J023, to ensure that also higher levels of EB3 expression had no detrimental effects. J030 showed overexpression effects and was not further used. MitoMice $^{15}$ (transgenic mice with CFP-labelled neuronal mitochondria to measure mitochondrial transport), Thy1:YFP-16 mice ${ }^{14}$ (which express cytoplasmic YFP in all motor axons) and $\triangle N L S$ - $W l d^{S}$ mice $^{38}$ (transgenic mice expressing the

'Wallerian degeneration slow' fusion protein carrying two-point mutations in a nuclear localization signal that redistribute the gene product to the cytoplasm; provided by M. Coleman, Babraham Institute, Cambridge, UK) were previously published. Mice overexpressing human superoxide dismutase 1 (wild type, G93A ${ }^{47}$ and $\mathrm{G} 85 \mathrm{R}^{48}$ mutations) were obtained from The Jackson Laboratory, Bar Harbor, USA. 'Preterminal' G85R animals had lost $10 \%$ of their peak body weight and were unable to sustain their body weight hanging from a grid for $>30 \mathrm{~s}^{39}$. Male and female animals were used, ranging in age from 6 to 18 weeks. All animal work conformed to institutional and national guidelines.

Single-cell reverse transciptase PCR. For collection of Purkinje cell cytoplasm we slightly modified a previously described protocol ${ }^{49}$. In brief, slices were placed in a recording chamber and perfused with artificial cerebrospinal fluid at room temperature. Purkinje cells were identified visually using an upright microscope (Eclipse E600FN; Nikon). Patch clamp pipettes were made from borosilicate glass $(2.0 / 0.3 \mathrm{~mm})$ using a Narishige PC-10 pipette puller. Tip diameters of about onethird of the cell soma diameter ( $8 \mu \mathrm{m}$ for Purkinje cells) were produced by modifying the heat of the second pulling step. The pipette solution consisted of $4 \mu \mathrm{l}$ $\mathrm{H}_{2} \mathrm{O}$ and $1 \mu \mathrm{l}$ M-MLV reverse transcriptase $5 \times$ reaction buffer ('First Strand Buffer', FSB; Promega). Under visual control, the tip of the pipette was gently attached to the selected cell and the soma sucked in. Under a dissecting microscope, the content of the pipette was pressure-ejected into a $0.5 \mathrm{ml}$ reaction tube and frozen at $-80^{\circ} \mathrm{C}$. Reverse transcription was performed as follows: The collected material was complemented with 'Master Mix 1' (1 $\mu$ l 1\% Nonidet P-40 and $1 \mu \mathrm{l} 5 \mathrm{mM}$ random primers $\mathrm{p}(\mathrm{dN}) 6$, Roche; $1 \mu \mathrm{l} 10 \mathrm{mM}$ of each dNTPs, $1 \mu \mathrm{l}$ FSB and $1 \mu \mathrm{l}$ RNasin plus; Promega), incubated at $70^{\circ} \mathrm{C}$ and then at $0{ }^{\circ} \mathrm{C}, 5 \mathrm{~min}$ each. Ten microlitres of 'Master Mix $2^{\prime}$ ( $2 \mu \mathrm{l} \mathrm{FSB}, 6.5 \mu \mathrm{l} \mathrm{H} 2 \mathrm{O}$ and $1.5 \mu \mathrm{l} \mathrm{M-MLV}$ reverse transcriptase; Promega) were added, followed by incubation for $1-2 \mathrm{~h}$ at $37^{\circ} \mathrm{C}$. The resulting cDNA was purified using silica matrix and stored at $-80^{\circ} \mathrm{C}$. For the comparison of expression levels in individual cells, $80 \%$ of cDNA material of a single cell was used. Rapid cycle PCR reactions performed in $20 \mu$ reactions in glass capillaries according to the manufacturer's instructions using the LightCycler FastStart DNA Master SYBR Green I kit (Roche). The amount of primer used per reaction was $10 \mathrm{pmol}$, the concentration of $\mathrm{MgCl} 2$ was $3 \mathrm{mM}$. Rapid cycle reverse transciptase PCR was performed on the LightCycler (Roche) and analysed with the LightCycler analysis software (version 3.5.3) in the log-linear phase using the so called 'fit point' method ${ }^{49}$.

Cell culture. Dorsal root ganglia were cultured following a published protocol ${ }^{50}$ Briefly, the ganglia from L4, L5 and L6 spinal levels of adult ( $>2$ month old) Thy1:EB3-YFP transgenic mice were dissected and incubated at $37^{\circ} \mathrm{C}$ in $3 \%$ collagenase type I $\left(3,000 \mathrm{U} \mathrm{ml}^{-1}\right.$, Worthington Biochemical) for $45 \mathrm{~min}$. The ganglia were then digested at $37^{\circ} \mathrm{C}$ with $0.25 \%$ trypsin (Roche) for $15 \mathrm{~min}$. By adding DMEM $/ 10 \%$ horse serum, the reaction was stopped, and cells collected by centrifugation at 700 r.p.m. for 5 min. Cells were resuspended in NeuroBasal media complemented with B-27 supplement, L-glutamine and penicillin-streptomycin (all Invitrogen) and plated on poly-L-lysine- (PLL; $1 \mathrm{mg} \mathrm{ml}^{-1}$, Sigma) and laminin $\left(5 \mu \mathrm{g} \mathrm{ml}^{-1}\right.$, Roche)-coated glass coverslips or glass-bottom dishes (MatTek). Cells were kept at $37^{\circ} \mathrm{C}$ in a humidified atmosphere containing $5 \% \mathrm{CO} 2$.

Primary hippocampal neurons derived from embryonic (E17) C57BL/6JCrl wild type (Charles River) or Thyl:EB3-YFP transgenic mice were cultured as established in previous studies ${ }^{51}$. In brief, hippocampi were dissected, digested with $0.05 \%$ trypsin-EDTA (Invitrogen) and washed with Hank's balanced salt solution. Afterwards cells were triturated using Pasteur pipettes and plated on PLL (Sigma)-coated coverslips or glass-bottom dishes in MEM/10\% horse serum. After $4 \mathrm{~h}$, media was changed to MEM containing N2 supplements.

Neurons were transfected using the Nucleofector system (Amaxa). The EB3mCherry and EB3-GFP plasmids were purified using the EndoFree Maxiprep Kit, (Qiagen) and used at a concentration of $3 \mu \mathrm{g}$. Microtubule dynamics of either Thy1:EB3-YFP transgenic or EB3-mCherry/-GFP-transfected wild-type cells were analysed $8 \mathrm{~h}$ or $24 \mathrm{~h}$ after plating. To this end, fluorescent images with a pixel size of $0.106 \mu \mathrm{m}$ were acquired in 3-s intervals for $3 \mathrm{~min}$ using a DeltaVision RT 
setup. Videos with 7 frames per second were processed using the open-source software, ImageJ/Fiji.

Tissue preparation, immunohistochemistry and microscopy. For tissue analysis, mice were transcardially perfused with $4 \%$ paraformaldehyde (PFA) diluted in $0.01 \mathrm{M}$ phosphate-buffered saline $(1 \times \mathrm{PBS})$ and post fixed for $24 \mathrm{~h}$ in $4 \% \mathrm{PFA}$ in $1 \times$ PBS. Following dissection, the tissue of interest (brain, spinal cord and eye) was either prepared for cryo-sectioning by incubation in $30 \% \mathrm{w} / \mathrm{v}$ sucrose (Sigma) in $1 \times$ PBS or vibratome sectioned. Sections (thickness: $30 \mu \mathrm{m}$ for cryostat, $100 \mu \mathrm{m}$ for vibratome) were stained with Neurotrace 594 (Molecular Probes, diluted 1:500 in $1 \times$ PBS) to label cell bodies and nuclei. Sections were mounted in Vectashield (Vector Laboratories) and image stacks were recorded using an FV1000 confocal microscope (Olympus) equipped with $\times 20 / 0.8$ N.A. and $\times 60 / 1.42$ N.A. oilimmersion objectives. Images were processed using ImageJ/Fiji and Adobe Photoshop.

Triangularis sterni muscles were fixed in methanol $\left(-20^{\circ} \mathrm{C}\right)$ for $10 \mathrm{~min}$, and then for $1 \mathrm{~h}$ in $4 \%$ PFA, stained with Alexa594-conjugated $\alpha$-Bungarotoxin (Invitrogen, $50 \mathrm{mg} \mathrm{\mu l}^{-1}$ diluted 1:50 in 1× PBS) and Alexa647-conjugated

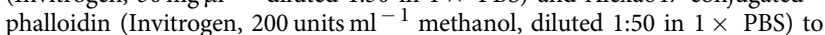
visualize acetylcholine receptors at the neuromuscular junction or muscle fibres, respectively, and analysed by confocal microscopy for parameters of neuromuscular health. Some muscles were stained with tubulin antibodies (Alexa555-conjugated mouse anti- $\beta$-tubulin, BD Pharmingen; anti-glu- $\alpha$-tubulin mouse monoclonal, Synaptic Systems) using standard protocols.

Electron microscopy. Animals were perfused transcardially with $2.5 \%$ glutaraldehyde and $2 \%$ PFA in $0.1 \mathrm{M}$ PBS. After post fixation for $24 \mathrm{~h}$, nerves were isolated and stained with $1 \%$ osmium tetroxide reduced in $1.5 \%$ potassium ferrocyanide. Tissues were washed in four changes of $0.1 \mathrm{M}$ sodium cacodylate buffer, dehydrated in an ascending ethanol series and two changes of $100 \%$ propylene oxide, infiltrated with Araldite 502/EMbed812 resin (Electron Microscopy Sciences), and finally polymerized at $60^{\circ} \mathrm{C}$ for $48 \mathrm{~h}$. Tissue blocks were oriented to cut either cross or lateral ultrathin sections $(\sim 60 \mathrm{~nm})$. Sections were collected on pioloform-coated slot grids and stained with $2 \%$ uranyl acetate and Reynold's lead citrate. Electron micrographs were obtained with a JEM-1400 transmission electron microscope (JEOL) operating at $120 \mathrm{kV}$. Micrographs were collected with an Ultrascan 1000 camera (Gatan) at a resolution of $1.6 \mathrm{~nm}$ per pixel.

Imaging microtubule dynamics ex vivo and in vivo. For the triangularis sterni explant, mice were lethally anesthetized with isoflurane and the anterior thoracic wall (with the attached triangularis sterni muscle and its innervating intercostal nerves) was isolated by cutting the ribs close to the vertebral column. The explant was pinned down on a Sylgard-coated $3.5 \mathrm{~cm}$ plastic Petri dish using minutien pins (Fine Science Tools). After excision, explants were kept in $95 \% \mathrm{O}_{2} / 5 \% \mathrm{CO}_{2}$ bubbled Ringer's solution ( $125 \mathrm{mM} \mathrm{NaCl}, 2.5 \mathrm{mM} \mathrm{KCl}, 1.25 \mathrm{mM} \mathrm{NaH}_{2} \mathrm{PO}_{4}, 26 \mathrm{mM}$ $\mathrm{NaHCO}_{3}, 2 \mathrm{mM} \mathrm{CaCl}, 1 \mathrm{mM} \mathrm{MgCl}, 20 \mathrm{mM}$ glucose). During imaging, explants were kept on a heated stage $\left(33-36^{\circ} \mathrm{C}\right.$; in this range, comet density varied by less than $20 \%$; data not shown) with a slow and steady flow of warmed and oxygenated Ringer's solution ${ }^{19}$. 'Proximal' are recording sites in the intercostal nerve proper; 'distal' are sites beyond the entry point of axons into the muscle. For in vivo imaging, the sciatic nerve was exposed in anesthetized mice ( $1.5 \%$ ketamine, $0.1 \%$ $\mathrm{vol} / \mathrm{vol}$ xylazine intraperitoneally) as described previously ${ }^{15}$, and imaged as described below. Animals were euthanized at the end of the experiment by cervical dislocation in deep anaesthesia.

Dynamic microtubules were imaged using an Olympus BX51WI microscope equipped with $\times 20 / 0.5$ N.A. and $\times 100 / 1.0$ N.A. water-immersion dipping-cone objectives, an automated filter wheel (Sutter) and a cooled CCD camera (Retiga EXi; Qimaging), controlled by $\mu$ Manager ${ }^{52}$. Neutral density and infrared-blocking filters in the light path were used to prevent photo toxicity and photo bleaching. To follow EB3 comets, 200 images per movie were acquired at $0.5 \mathrm{~Hz}(2 \mathrm{~Hz}$ for analysis of comet track parameters) using an exposure time of $500 \mathrm{~ms}$. For measuring paclitaxel effects, the drug was included in the superfusion solution (solvent, $0.2 \%$ dimethylsulphoxide) and comet densities were recorded repeatedly for up to $3 \mathrm{~h}$. For analysis of degenerating and regenerating axons, mice were anesthetized with ketamine-xylazine (as above) and intercostal nerves were transected at the third $\mathrm{rib}^{53}$. After surgical closure of the wound, the mice were placed in a heated recovery chamber. Explants were prepared as described above after the desired interval. To image alterations in microtubule dynamics after acute axon cut, we performed laser axotomies in the triangularis sterni explant using an FV1000MPE two-photon microscope (Olympus). A femtosecond pulsed Ti:Sapphire laser (Mai Tai HP, Newport/Spectra Physics) tuned to $910 \mathrm{~nm}$ was placed on the axon for $3-5 \mathrm{~s}$ at $100 \%$ power using a $\times 100 / 1.0$ N.A. water objective. The explants were then transferred to a wide-field setup and imaged as described above. To assay the effects of paclitaxel treatment on $\mathrm{AAD}$, explants were pretreated for $1.5 \mathrm{~h}$ before laser axotomy.

Time-lapse imaging in acute cerebellar slices. To study microtubule dynamics in Purkinje cell dendrites, acute cerebellar slices of Thy1:EB3-YFP transgenic mice were prepared. Mice were lethally anesthetized with $\mathrm{CO}_{2}$ and the cerebellum was rapidly removed and placed into oxygenated Ringer's solution at $0-2{ }^{\circ} \mathrm{C}$. Slices (300 $\mu \mathrm{m}$ thick) were cut using a vibratome slicer (Leica). After cutting, slices were kept at $34^{\circ} \mathrm{C}$ for $1 \mathrm{~h}$ and then imaged for up to $4 \mathrm{~h}$ at $33-36^{\circ} \mathrm{C}$ in oxygenated Ringer's solution. Slices were imaged on an FV1000 confocal microscope (Olympus) using $\times 20 / 0.5$ N.A. and $\times 100 / 1.0$ N.A. water-immersion dipping-cone objectives.

Imaging of microtubule dynamics in the spinal cord. In vivo imaging of the lumbar spinal cord was carried out as previously described ${ }^{54,55}$. Briefly, mice were anaesthetized by an intraperitoneal injection of ketamine-xylazine (ketamine $87 \mathrm{mg} \mathrm{kg}^{-1}$, xylazine $\left.13 \mathrm{mg} \mathrm{kg}^{-1}\right)$. Anesthesia was reapplied as needed (60$120 \mathrm{~min}$ ). To access the dorsal surface of the lumbar spinal cord, a laminectomy was performed. To minimize movement during high-resolution imaging, the vertebral column was immobilized using compact spinal cord clamps (Narishige) ${ }^{56}$ and mice were intubated following a tracheotomy. Symmetrical incisions were made lateral to the spinal column, one pair along the vertebral level rostral, and another pair two segments caudal to the laminectomy site for insertion of the spinal cord clamps. To allow for the formation of a liquid reservoir (regularly filled with prewarmed mouse artificial cerebrospinal fluid; aCSF: $148.2 \mathrm{NaCl}, 3.0 \mathrm{KCl}, 1.4$ $\mathrm{CaCl} 2,0.8 \mathrm{MgCl} 2,0.8 \mathrm{Na} 2 \mathrm{HPO} 4,0.2 \mathrm{NaH} 2 \mathrm{PO} 4$ in $\mathrm{mM}$ ) and access with a water dipping-cone objective $(\times 25 / 1.05)$, an agarose well $(2 \%$ agarose in aCSF $)$ was made around the surgery site. Time-lapse recordings were acquired using an Olympus FVMPE-RS two-photon system equipped with a femtosecond pulsed Ti:Sapphire laser (Mai Tai HP, Newport/Spectra Physics). The laser was tuned to $930 \mathrm{~nm}$ for excitation of YFP. Detectors consisted of gallium arsenide phosphide photomultiplier tubes. All light was first filtered through a $690 \mathrm{~nm}$ short pass dichroic mirror. A $495-540 \mathrm{~nm}$ barrier filter in front of the gallium arsenide phosphide detectors was used for imaging YFP.

Cranial window implantation and cortical in vivo imaging. Cranial window implantation was performed as previously described ${ }^{23,24}$. In brief, mice were anesthetized with an intraperitoneal injection of ketamine-xylazine (ketamine $140 \mathrm{mg} \mathrm{kg}^{-1}$, xylazine $\left.10 \mathrm{mg} \mathrm{kg}^{-1}\right)$. In addition, dexamethasone $\left(6 \mathrm{mg} \mathrm{kg}^{-1}\right)$ was injected intraperitoneally to prevent the development of cerebral oedema ${ }^{24}$. Mice were then placed on a heating blanket and stabilized in a stereotaxic frame. Using a dental drill (Schick-Technikmaster C1; Pluraden), a round craniotomy was made and immediately covered with round coverslip $(3 \mathrm{~mm})$. The coverslip was sealed to the skull using dental acrylic (Cyano-Veneer fast; Schein). A small metal bar was cemented next to the coverslip to allow repositioning of the mouse during subsequent imaging sessions. After surgery, mice received subcutaneous doses of the analgesic Carprofen $\left(7.5 \mathrm{mg} \mathrm{kg}^{-1}\right)$ and the antibiotic cefotaxime $\left(5 \mathrm{mg} \mathrm{kg}^{-1}\right)$. Imaging started 21 days after surgery to allow mice to recover and cranial windows to become clear. For time-lapse imaging of the somatosensory cortex, animals were anesthetized as described above and placed into a metal holder. Movies were acquired using the Olympus FVMPE-RS system as described for spinal cord imaging

Image analysis and processing. To quantify comet parameters, images were analysed using the open-source software ImageJ/Fiji (http://fiji.sc) and the obtained movies were auto-aligned using the 'StackReg' algorithm ${ }^{57}$. Dynamic microtubules were analysed with the MTrackJ-plugin (developed by E. Meijering, Biomedical Imaging Group, Erasmus Medical Center, Rotterdam) by manual tracking of fluorescent EB3 comets that appeared on at least three consecutive frames. Polar plots were generated with IGOR software (WaveMetrics). Time-lapse movies recorded on scanning microscopes were de-noised using the CANDLE algorithm, a Matlab based software tool ${ }^{58}$

The following parameters were measured: orientation (angle of comet deviation measured from the local neurite orientation set to $0^{\circ}$ ), density of dynamic microtubules (average number of moving fluorescent comets in 200 frames in a random $>50 \mu \mathrm{m}^{2}$ axon area expressed as comets $\mu \mathrm{m}^{-2}$ ) and average speed of EB3 comets (total displacement divided by observation time). For analysis of comet track parameters, movies at higher temporal resolution were obtained. 'Pauses' were identified as intervals during which the comet translocated less than 2 pixels (approximately the 'jitter' of our manual tracking; often the comet briefly dimmed or disappeared but resumed growth from the same position); 'set-backs' were phases, during which comets briefly disappeared and then reappeared further proximal along the same trajectory.

A number of reasons prompted us to analyse comet density in two dimensions, although a full three-dimensional treatment seems more obvious at first glance. First, already when imaging in a single plane, recordings tend to be limited by temporal resolution and signal-to-noise constraints; three-dimensional analysis would result in severe under-sampling of comets with most microscope technologies. Furthermore, based on an estimate of the depth of field of our objectives, we believe that in most cases we capture comets in a subvolume of the relatively thick neurites that we imaged. Indeed, comet density did not correlate with neurite thickness or medial-to-lateral position within motor axons, as would be expected if a full cylinder was sampled (data not shown). As the depth of field can, however, not easily be measured, and in some thin neurites (for example, cortical dendrites) the above listed considerations argue that a certain volume error persists (which leads to an underestimate of volumetric comet density), we felt it to 
be more appropriate to restrict our analysis to area densities to be upfront about the limitations in the quantitative accuracy of our analysis.

To quantify morphological features of neuromuscular junctions, confocal image stacks of fixed triangularis sterni explants from EB3-YFP-positive and -negative litter mates were analysed as follows. The size of neuromuscular junctions was determined by measurements of the thresholded area of Bungarotoxin staining on confocal stacks. The size of the muscle fibres was measured on rotated confocal projections as the circumference of individual fibres revealed by phalloidin staining.

To measure the ratio of detyrosinated versus total tubulin, confocal images of antibody stainings were analysed in Image/Fiji. A region of interest was placed within an axon of Thy1:EB3-YFP transgenic mice and EB3-YFP-negative litter mates. Both channels were background subtracted and their ratio was determined in a single optical section.

We determined the number of anterogradely and retrogradely transported mitochondria ('transport flux') in explants from MitoMice (line C) crossed to heterozygous Thy1:EB3-YFP mice as the number of fluorescent mitochondria per minute that crossed a vertical line placed across the axon. For this purpose, timelapse movies were obtained as described for imaging microtubule dynamics and results from transgenic and non-transgenic litter mates were compared.

Electron micrographs were analysed using Image//Fiji. Microtubule length and orientation were analysed on lateral sections of $25 \mu \mathrm{m}$ axon segments where orientation angle of $0^{\circ}$ was considered parallel to the axial direction of the axon. Microtubule density was analysed on cross-sections of random axons ranging between $\sim 4-50 \mu \mathrm{m}^{2}$.

For image representation, confocal and wide-field images were combined after pseudocolouring using the 'screen' function in Photoshop and gamma was adjusted non-linearly in some images that illustrate qualitative properties to better reveal low-intensity objects. For overview images of expression patterns, multiple stitched frames were montaged in Photoshop. Montages of tiled confocal images are presented on a black background.

Critical comparative data sets (for example, effects of paclitaxel on AAD and comet densities in SOD mice) were analysed by one or two blinded researchers.

Statistics. Sample sizes were determined on the basis of prior experience in previous experiments. Statistics were performed using Microsoft Excel and GraphPad PRISM software. Statistical significance was determined using two-tailed $t$-tests or analysis of variance where appropriate. $P$ values $<0.05$ were considered to be significant and indicated by (*); $P$ values $<0.01$ were indicated by ${ }^{(* *)}$ and $<0.001$ by '***). Graphs show mean \pm s.e.m. Sample sizes in the text are given as $n / m=n$ neurites from $m$ mice, unless stated otherwise.

\section{References}

1. Conde, C. \& Caceres, A. Microtubule assembly, organization and dynamics in axons and dendrites. Nat. Rev. Neurosci. 10, 319-332 (2009).

2. Mitchison, T. \& Kirschner, M. Dynamic instability of microtubule growth. Nature 312, 237-242 (1984).

3. Neukirchen, D. \& Bradke, F. Neuronal polarization and the cytoskeleton. Dev. Biol. 22, 825-833 (2011).

4. Bishop, D. L., Misgeld, T., Walsh, M. K., Gan, W. B. \& Lichtman, J. W. Axon branch removal at developing synapses by axosome shedding. Neuron 44, 651-661 (2004)

5. Chevalier-Larsen, E. \& Holzbaur, E. L. Axonal transport and neurodegenerative disease. Biochim. Biophys. Acta 1762, 1094-1108 (2006).

6. Fanara, P. et al. Stabilization of hyperdynamic microtubules is neuroprotective in amyotrophic lateral sclerosis. J. Biol. Chem. 282, 23465-23472 (2007).

7. d'Ydewalle, C. et al. HDAC6 inhibitors reverse axonal loss in a mouse model of mutant HSPB1-induced Charcot-Marie-Tooth disease. Nat. Med. 17, 968-974 (2011).

8. Galjart, N. Plus-end-tracking proteins and their interactions at microtubule ends. Curr. Biol. 20, R528-R537 (2010).

9. Akhmanova, A. \& Hoogenraad, C. C. Microtubule plus-end-tracking proteins: mechanisms and functions. Curr. Opin. Cell Biol. 17, 47-54 (2005).

10. Stepanova, T. et al. Visualization of microtubule growth in cultured neurons via the use of EB3-GFP (end-binding protein 3-green fluorescent protein). J. Neurosci. 23, 2655-2664 (2003).

11. Erez, H. et al. Formation of microtubule-based traps controls the sorting and concentration of vesicles to restricted sites of regenerating neurons after axotomy. J. Cell Biol. 176, 497-507 (2007).

12. Stone, M. C., Roegiers, F. \& Rolls, M. M. Microtubules have opposite orientation in axons and dendrites of Drosophila neurons. Mol. Biol. Cell 19, 4122-4129 (2008).

13. Norden, C., Young, S., Link, B. A. \& Harris, W. A. Actomyosin is the main driver of interkinetic nuclear migration in the retina. Cell 138, 1195-1208 (2009).

14. Feng, G. et al. Imaging neuronal subsets in transgenic mice expressing multiple spectral variants of GFP. Neuron 28, 41-51 (2000).

15. Misgeld, T., Kerschensteiner, M., Bareyre, F. M., Burgess, R. W. \& Lichtman, J. $\mathrm{W}$. Imaging axonal transport of mitochondria in vivo. Nat. Methods 4, 559-561 (2007)
16. De Paola, V., Arber, S. \& Caroni, P. AMPA receptors regulate dynamic equilibrium of presynaptic terminals in mature hippocampal networks. Nat. Neurosci. 6, 491-500 (2003).

17. Wyatt, R. M. \& Balice-Gordon, R. J. Heterogeneity in synaptic vesicle release at neuromuscular synapses of mice expressing synaptopHluorin. J. Neurosci. 28, 325-335 (2008)

18. Komarova, Y. et al. EB1 and EB3 control CLIP dissociation from the ends of growing microtubules. Mol. Biol. Cell 16, 5334-5345 (2005).

19. Kerschensteiner, M., Reuter, M. S., Lichtman, J. W. \& Misgeld, T. Ex vivo imaging of motor axon dynamics in murine triangularis sterni explants. Nat. Protoc. 3, 1645-1653 (2008).

20. Applegate, K. T. et al. plusTipTracker: quantitative image analysis software for the measurement of microtubule dynamics. J. Struct. Biol. 176, 168-184 (2011).

21. Nicovich, P. R. \& Zhou, F. Q. Acquisition frame rate affects microtubule plusend tracking analysis. Nat. Methods 11, 219-220 (2014).

22. Baas, P. W., Deitch, J. S., Black, M. M. \& Banker, G. A. Polarity orientation of microtubules in hippocampal neurons: uniformity in the axon and nonuniformity in the dendrite. Proc. Natl Acad. Sci. USA 85, 8335-8339 (1988).

23. Fuhrmann, M., Mitteregger, G., Kretzschmar, H. \& Herms, J. Dendritic pathology in prion disease starts at the synaptic spine. J. Neurosci. 27, 6224-6233 (2007).

24. Holtmaat, A. et al. Long-term, high-resolution imaging in the mouse neocortex through a chronic cranial window. Nat. Protoc. 4, 1128-1144 (2009).

25. Schiff, P. B. \& Horwitz, S. B. Taxol stabilizes microtubules in mouse fibroblast cells. Proc. Natl Acad. Sci. USA 77, 1561-1565 (1980).

26. Jordan, M. A. \& Wilson, L. Microtubules as a target for anticancer drugs. Nat. Rev. Cancer 4, 253-265 (2004).

27. Erturk, A., Hellal, F., Enes, J. \& Bradke, F. Disorganized microtubules underlie the formation of retraction bulbs and the failure of axonal regeneration. J. Neurosci. 27, 9169-9180 (2007).

28. Hellal, F. et al. Microtubule stabilization reduces scarring and causes axon regeneration after spinal cord injury. Science 331, 928-931 (2011).

29. Barten, D. M. et al. Hyperdynamic microtubules, cognitive deficits, and pathology are improved in tau transgenic mice with low doses of the microtubule-stabilizing agent BMS-241027. J. Neurosci. 32, 7137-7145 (2012)

30. Kerschensteiner, M., Schwab, M. E., Lichtman, J. W. \& Misgeld, T. In vivo imaging of axonal degeneration and regeneration in the injured spinal cord. Nat. Med. 11, 572-577 (2005).

31. Chen, L., Stone, M. C., Tao, J. \& Rolls, M. M. Axon injury and stress trigger a microtubule-based neuroprotective pathway. Proc. Natl Acad. Sci. USA 109, 11842-11847 (2012).

32. El Bejjani, R. \& Hammarlund, M. Neural regeneration in Caenorhabditis elegans. Annu. Rev. Genet. 46, 499-513 (2012).

33. Tanaka, E. M. \& Ferretti, P. Considering the evolution of regeneration in the central nervous system. Nat. Rev. Neurosci. 10, 713-723 (2009).

34. Galbraith, J. A. \& Terasaki, M. Controlled damage in thick specimens by multiphoton excitation. Mol. Biol. Cell 14, 1808-1817 (2003).

35. Stone, M. C., Nguyen, M. M., Tao, J., Allender, D. L. \& Rolls, M. M. Global upregulation of microtubule dynamics and polarity reversal during regeneration of an axon from a dendrite. Mol. Biol. Cell 21, 767-777 (2010).

36. Nguyen, Q. T., Sanes, J. R. \& Lichtman, J. W. Pre-existing pathways promote precise projection patterns. Nat. Neurosci. 5, 861-867 (2002).

37. Coleman, M. P. \& Freeman, M. R. Wallerian degeneration, wld(s), and nmnat Annu. Rev. Neurosci. 33, 245-267 (2010).

38. Beirowski, B. et al. Non-nuclear Wld(S) determines its neuroprotective efficacy for axons and synapses in vivo. J. Neurosci. 29, 653-668 (2009).

39. Marinkovic, P. et al. Axonal transport deficits and degeneration can evolve independently in mouse models of amyotrophic lateral sclerosis. Proc. Natl Acad. Sci. USA 109, 4296-4301 (2012).

40. Jaworski, J. et al. Dynamic microtubules regulate dendritic spine morphology and synaptic plasticity. Neuron 61, 85-100 (2009).

41. Holtmaat, A. \& Svoboda, K. Experience-dependent structural synaptic plasticity in the mammalian brain. Nat. Rev. Neurosci. 10, 647-658 (2009).

42. Lichtman, J. W. \& Colman, H. Synapse elimination and indelible memory. Neuron 25, 269-278 (2000).

43. Bridge, K. E. et al. Late onset distal axonal swelling in YFP-H transgenic mice. Neurobiol. Aging 30, 309-321 (2009).

44. Baas, P. W. \& Lin, S. Hooks and comets: the story of microtubule polarity orientation in the neuron. Dev. Neurobiol. 71, 403-418 (2011).

45. Marinkovic, P., Godinho, L. \& Misgeld, T. in Imaging In Neuroscience And Development. (eds Yuste, R. \& Konnerth, A.) Chapter 22 (CSH Press, 2011).

46. Caroni, P. Overexpression of growth-associated proteins in the neurons of adult transgenic mice. J. Neurosci. Methods 71, 3-9 (1997).

47. Gurney, M. E. et al. Motor neuron degeneration in mice that express a human $\mathrm{Cu}, \mathrm{Zn}$ superoxide dismutase mutation. Science 264, 1772-1775 (1994).

48. Bruijn, L. I. et al. Elevated free nitrotyrosine levels, but not protein-bound nitrotyrosine or hydroxyl radicals, throughout amyotrophic lateral sclerosis (ALS)-like disease implicate tyrosine nitration as an aberrant in vivo property of 
one familial ALS-linked superoxide dismutase 1 mutant. Proc. Natl Acad. Sci. USA 94, 7606-7611 (1997).

49. Durand, G. M., Marandi, N., Herberger, S. D., Blum, R. \& Konnerth, A. Quantitative single-cell RT-PCR and $\mathrm{Ca} 2+$ imaging in brain slices. Pflugers Arch. 451, 716-726 (2006).

50. Enes, J. et al. Electrical activity suppresses axon growth through $\mathrm{Ca}(\mathrm{v}) 1.2$ channels in adult primary sensory neurons. Curr. Biol. 20, 1154-1164 (2010).

51. Witte, H., Neukirchen, D. \& Bradke, F. Microtubule stabilization specifies initial neuronal polarization. J. Cell Biol. 180, 619-632 (2008).

52. Edelstein, A., Amodaj, N., Hoover, K., Vale, R. \& Stuurman, N. Computer control of microscopes using microManager. Curr. Protoc. Mol. Biol Chapter 14, 20 (2010).

53. Angaut-Petit, D. \& Faille, L. Inability of regenerating mouse motor axons to innervate a denervated target. Neurosci. Lett. 75, 163-168 (1987).

54. Nikic, I. et al. A reversible form of axon damage in experimental autoimmune encephalomyelitis and multiple sclerosis. Nat. Med. 17, 495-499 (2011).

55. Romanelli, E. et al. Cellular, subcellular and functional in vivo labeling of the spinal cord using vital dyes. Nat. Protoc. 8, 481-490 (2013).

56. Davalos, D. et al. Stable in vivo imaging of densely populated glia, axons and blood vessels in the mouse spinal cord using two-photon microscopy. J. Neurosci. Methods 169, 1-7 (2008).

57. Thevenaz, P., Ruttimann, U. E. \& Unser, M. A pyramid approach to subpixel registration based on intensity. IEEE Trans. Image Process 7, 27-41 (1998).

58. Coupe, P., Munz, M., Manjon, J. V., Ruthazer, E. S. \& Collins, D. L. A CANDLE for a deeper in vivo insight. Med. Image Anal. 16, 849-864 (2012).

\section{Acknowledgements}

We would like to thank Manuela Budak, Ljiljana Marinković and Nebahat Budak for animal husbandry; Sarah Bechtold, Yvonne Hufnagel and Kristina Wullimann for technical assistance, Monika Schetterer for administrative support and Gabriela Plucińska for help with initially testing the EB3-YFP construct in zebrafish. We thank Michael Coleman (Babraham Institute) for the $\Delta N L S$-Wld $d^{S}$ mice and Brian Link (Medical College of Wisconsin) for the EB3-GFP construct. We thank Ed Ruthazer for point out the CANDLE algorithm to us. T.M., P.M., J.H. and F.B. are supported by the German Center for Neurodegenerative Disease (DZNE). Further support came from the Deutsche Forschungsgemeinschaft (DFG) via the Center for Integrated Protein Science Munich (EXC 114; A.K., T.M.), the Munich Cluster for Systems Neurology (EXC 1010 SyNergy; J.H., A.K., M.K., T.M.), Transregio 128 (M.K.), Priority Programme 1710 (M.K., T.M.), Research Training Group 1373 (P.E.), Collaborative Research Center 870 (A.K., L.G., T.M.) and Sonderforschungsbereich 1191 (F.B.). M.K.'s laboratory is further financed by the German Federal Ministry of Research and Education (BMBF;
Competence Network Multiple Sclerosis), the European Research Council under the European Union's Seventh Framework Programme (FP/2007-2013; ERC Grant Agreement n. 310932), the Hertie-Foundation and the 'Verein Therapieforschung für MS-Kranke e.V.'. T.M. is supported by the European Research Council under the European Union's Seventh Framework Programme (FP/2007-2013; ERC Grant Agreement n. 616791). F. B. is also supported by the International Foundation for Research in Paraplegia and Wings for Life. Work on this project was further supported by the national funding agency (BMBF) in the frame of ERA-Net '2-photon imaging' (A.K., T.M.). P.R.W. was supported by the Human Frontier Science Program and the Wings for Life Foundation. D.B.'s lab is supported by the National Science Foundation (Award \#1126196). T.K. and P.M. were supported by the Graduate School of Technische Universität München (TUM-GS)

\section{Author contributions}

T.K., M.K., L.G, and T.M. conceived the experiments. T.K., P.R.W., P.E., J.H. and P.M. planned and performed time-lapse imaging experiments and image analysis. T.K., P.M., E.E.W. and D.B. prepared, imaged and evaluated electron microscopy specimens. S.S. and F.B. planned and performed cell culture experiments. T.K., J.H., R.M.K. and A.K. planned and performed single-cell PCR experiments. T.K., L.G. and R.N. generated and characterized the transgenic mice. T.K., M.K., D.B., L.G, and T.M. wrote the paper with input from all the authors.

\section{Additional information}

Supplementary Information accompanies this paper at http://www.nature.com/ naturecommunications

Competing financial interests: The authors declare no competing financial interests

Reprints and permission information is available online at http://npg.nature.com/ reprintsandpermissions/

How to cite this article: Kleele, T. et al. An assay to image neuronal microtubule dynamics in mice. Nat. Commun. 5:4827 doi: 10.1038/ncomms5827 (2014).

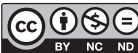

This work is licensed under a Creative Commons AttributionNonCommercial-NoDerivs 4.0 International License. The images or other third party material in this article are included in the article's Creative Commons license, unless indicated otherwise in the credit line; if the material is not included under the Creative Commons license, users will need to obtain permission from the license holder to reproduce the material. To view a copy of this license, visit http:// creativecommons.org/licenses/by-nc-nd/4.0/ 